\title{
MONITORING MICROMECHANICAL BUCKLING AT HIGH-SPEED FOR SENSING AND TRANSDUCER APPLICATIONS
}

\author{
Berke Demiralp ${ }^{1}$, Hadi S. Pisheh ${ }^{1}$, Berk Kucukoglu ${ }^{1}$, Utku Hatipoglu ${ }^{l}$, and M. Selim Hanay ${ }^{1}$ \\ ${ }^{1}$ The Department of Mechanical Engineering, and UNAM, Bilkent University, TURKEY
}

\begin{abstract}
Controlling the amount and direction of buckling at micro- and nano-scale efficiently opens up avenues for novel actuation and sensor applications. Earlier platforms that can achieve a full and non-thermal control of microscopic buckling operated only with a time resolution of $40 \mathrm{~ms}$. Here, we have measured the buckling amount of a beam starting from unbuckled position and reaching to large post-buckling deformations by collecting secondary electrons under scanning electron microscope. Line mode is used for ultrafast measurements with $33 \mathrm{kHz}$ scan frequency, and a displacement noise floor of $40 \mathrm{pm} / \sqrt{\mathrm{Hz}}$ was obtained. Moreover, by further reduction in the device dimensions, the buckling threshold voltage was reduced by a factor of three compared to similar platforms.
\end{abstract}

\section{KEYWORDS}

NEMS, Buckling, Post-Buckling, Nanofabrication, Scanning Electron Microscope Line Mode.

\section{INTRODUCTION}

Controllable nonlinear dynamics of micromechanical transducers bring forth novel functionalities such as large displacement amplitudes and the emergence of bistable states. As a nonlinearity mechanism, buckling transition offers many advantages, especially if buckling can be observed and controlled at high speed and with low displacement noise.

When a mechanical beam approaches and passes through the buckling transition, its effective spring constant shrinks, which results in large deflections to be attained by the application of a small amount of force. Moreover, the system dynamics post-buckling can be described as a double-well potential with two distinct states (Figure 1). The emergence of such bistable states enables one to store and operate upon information mechanically.

Earlier work with micro- and nano-mechanical buckling concentrated on either using thermally-induced buckling [1] or pre-buckled beams [2, 3]. These approaches, however, do not provide all the necessary knobs to fully control buckling mechanism, such as the depth of potential energy wells and asymmetry between the wells. Clearly, to transform a nonlinear mechanism into an engineering system, all critical parameters of the system should be controllable by the user. Recently [4], a full electrostatic control of nanomechanical buckling was introduced by integrating an inverted comb drive actuator with a nanomechanical beam (a similar platform is shown in Figure 2 here). While the voltage on the comb drive controlled the axial compression force, additional side gates on either side of the beam enabled for the adjustment of the asymmetry between the bistable states (Fig. 2). With this arrangement, the buckling system exhibited large displacement amplitudes, mechanical bit storage, and ratedependent hysteresis in this platform.
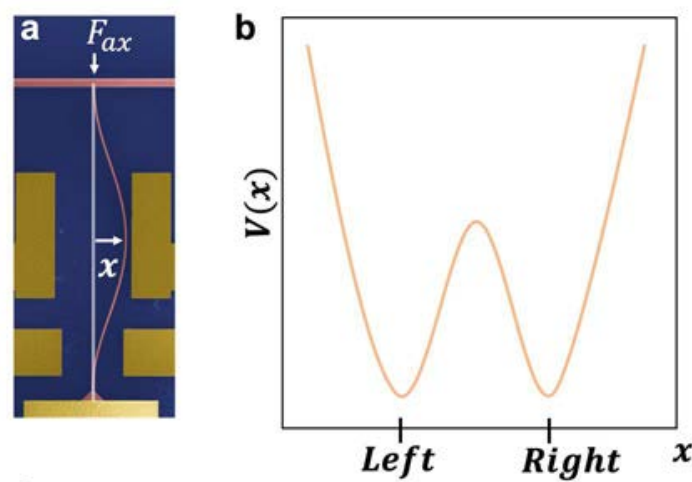

C

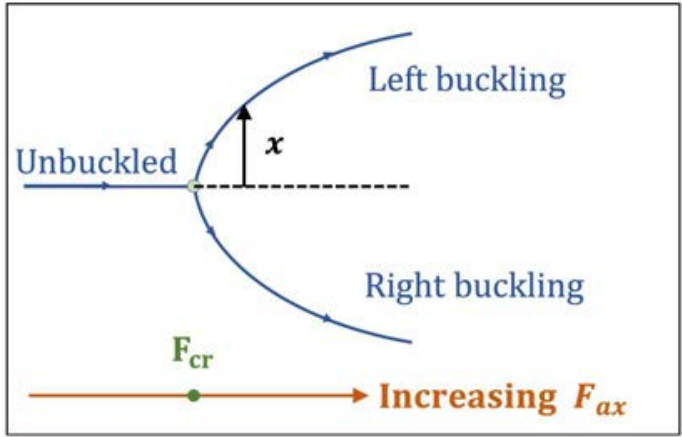

Figure 1: Buckling transition at the microscale. (a) The application of a sufficiently large axial force $F_{a x}$ can induce buckling and results in a displacement ( $x$ ) from the neutral axis which can reach a significant ratio of the beam length. (b) The potential energy (V) landscape of the system exhibits a double-well potential. Asymmetry between the wells can be induced by applying voltages to the side gates visible in part a. (c) Bifurcation diagram of the system showing a pitchfork bifurcation at critical Force, $F_{c r}$.

However, the first experiments with this platform [4] relied on the video mode, i.e. full-frame scan, of an SEM (Scanning Electron Microscope) which limited the measurement bandwidth of the beam displacement to video rates. Performing high speed measurements is extremely critical to unravel novel nonlinear dynamics such as the snap through transitions between the bistable states and the emergence of chaos. To increase the measurements speed, we have developed a protocol based on the line mode of SEM. While the use of SEM line mode directly speeded up the measurement rate to $33 \mathrm{kHz}$, it also introduced new challenges especially in terms of data processing. After resolving these challenges through a new readout strategy, we have obtained a displacement noise floor of $40 \mathrm{pm} / \sqrt{ } \mathrm{Hz}$. The measurement speed and displacement noise obtained in this work enables us to probe novel nonlinear dynamical effects at the micro/nano scale.

\section{NEMS BASED BUCKLING DEVICE}

The principle of the device is to buckle either to the left or right when compression force is applied to the beam. 
Buckling direction can be controlled by two side gates which are located $5 \mu \mathrm{m}$ away from the beam (Fig. 2b). Two sets of comb drive structures are present in the device with one being stationary and other being movable. When a voltage difference is applied to stationary and movable parts of the combs, movable one exerts a compression force on the beam and when that force exceeds the critical compression force, buckling occurs. Critical compression force is a function of material, voltage difference between combs, geometry of the beam and comb drive actuators.

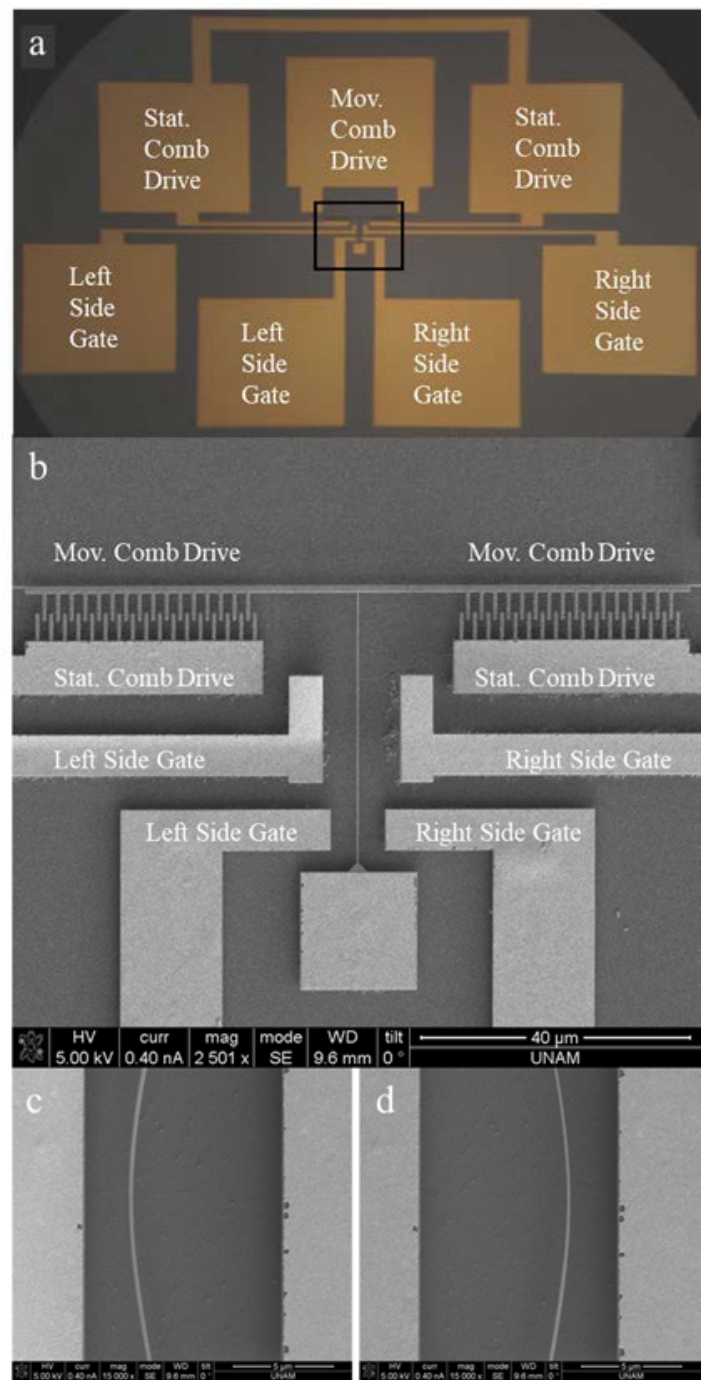

Figure 2: (a) General view of the pads. Pads belonging to stationary combs are connected to each other. (b) SEM image of the device, located at the black rectangle in (a). (c) Left buckling state. (d) Right buckling state.

Device fabrication is performed starting with Silicon on Insulator wafer with a composition of $250 \mathrm{~nm} \mathrm{Si}$ on top of $3 \mu \mathrm{m} \mathrm{SiO}{ }_{2}$ and with $650 \mu \mathrm{m}$ thick Si base. Starting with the standard cleaning procedure on the substrate, electron beam lithography (EBL) is performed using PMMA to define pads and electrical guides. Following the patterning, $120 \mathrm{~nm}$ of $\mathrm{Au}$ is deposited with thermal evaporation. The sample is left for acetone bath for lift-off about 12 hours. Then, a second EBL process is done using PMMA to define combs and beam. During this process, the features from the first EBL process is also defined to protect gold layers from the subsequent inductively coupled plasma procedure. Following the second EBL patterning, $100 \mathrm{~nm}$ of $\mathrm{SiO}_{2}$ is deposited using electron beam evaporator. After the lift-off process, again in acetone bath for 12 hours, the Si layer which is not covered with $\mathrm{SiO}_{2}$ is etched by a Cl-based plasma etching. For the last step, the device is exposed to hydrofluoric acid vapor to form suspended beam and comb drive actuators as well as to get rid of protective $\mathrm{SiO}_{2}$ mask.

\section{SEM LINE MODE MEASUREMENT}

The most common way to view a device under SEM is to perform a full frame scan where electrons start a scanning cycle from left to right, starting at the top and moving to a lower line subsequently after each cycle finishes. When line reaches the very bottom, it turns back to the very first position where scan started, as shown in Fig. 3. In addition to the full frame mode, SEM employs two other modes: line mode and spot mode. In the former only a single line is scanned and after the scan cycle is completed, electron line does not move to a bottom line rather it stays on the same selected line (Figure 3). In the latter, a circular area is scanned only. Our focus will be on the line mode for the context of this paper, since the position of the beam with respect to the two side gates can be extracted quickly and reliably in this mode.
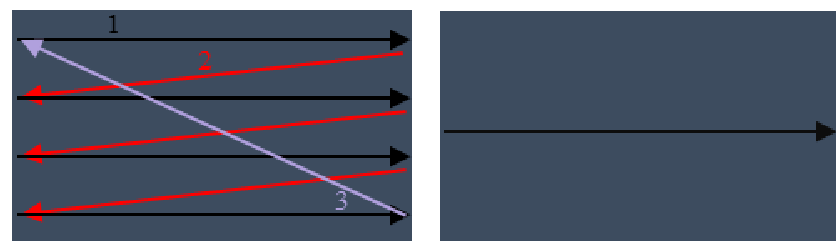

Figure 3: Full frame (left) vs line mode (right). In line mode, only a specific line of choice is scanned which is indicated by black arrow.

\section{EXPERIMENT}

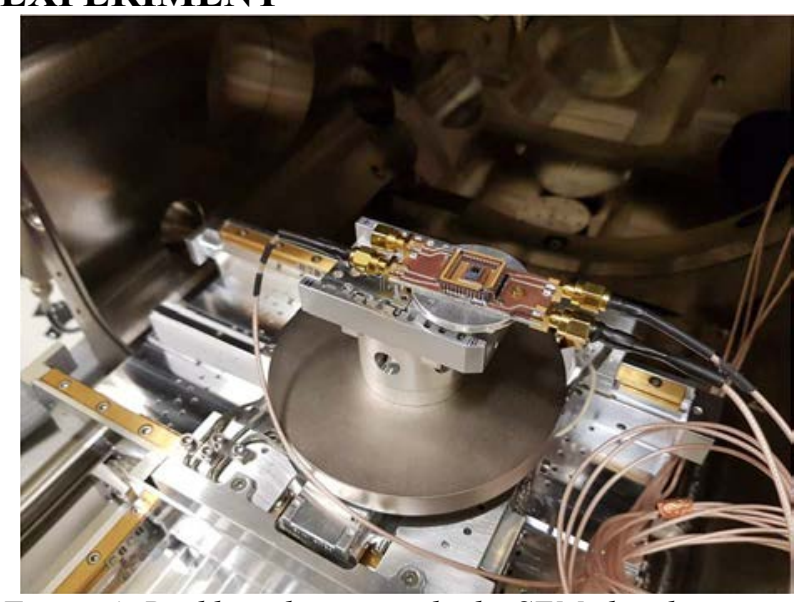

Figure 4: Buckling device inside the SEM chamber.

Experiments are conducted inside an SEM chamber; hence electrical connections are made by electronic feedthroughs. The buckling device is wire bonded to a PCB and the PCB is connected to a voltage supply (Rohde Schwarz HMP 2030) for actuating the comb drives and controlling the buckling direction. Secondary electrons collected using the line mode, which is available to the external user at an electrical feedthrough of the SEM, are 
converted to voltage values by a DAQ card (Compact RIO) for data analysis. A photo of the buckling device inside the SEM chamber is present at Fig. 4, and experiment schematic can be seen at Fig. 5.

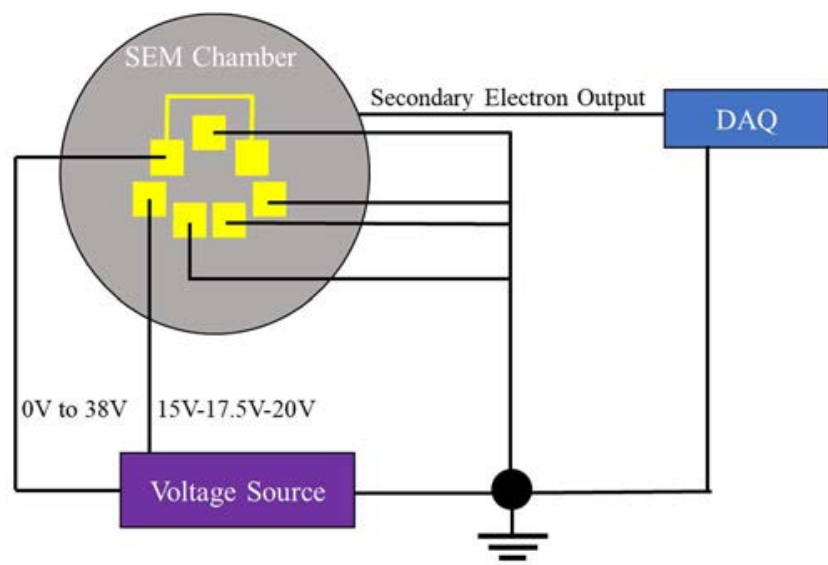

Figure 5: Experiment schematic. Non-ground connection functions are mentioned at their proximity.

A single line scan period is $30 \mu$ s and the data are acquired by Compact RIO with the rate of $200 \mathrm{kSa} / \mathrm{s}$. Electron gun voltage is fixed through the experiment and is equal to $5 \mathrm{kV}$. Starting from the unbuckled position of the beam, the left or right-side gate is turned on to $15 \mathrm{~V}$ and flexural deflection is measured before the onset of buckling (Figure 6). Afterwards, the comb voltage difference (controlling the axial compression) is increased starting from $0 \mathrm{~V}$ to $38 \mathrm{~V}$ by $0.2 \mathrm{~V}$ steps by applying voltage only to the stationary combs. For each voltage step, the deflection data is acquired. During the entire experiment, the beam voltage is at ground level and there is no voltage applied to the movable combs since they are connected to each other. Also, other side gates are at the ground level as well. After voltage scan from $0 \mathrm{~V}$ to $38 \mathrm{~V}$, beam is brought back to the unbuckled position and experiment is conducted for $17.5 \mathrm{~V}$ and $20 \mathrm{~V}$ side gate voltages.

\section{DATA ANALYSIS AND RESULTS}

After one set of experiment for one fixed side gate, approximately $65 \mathrm{M}$ data points are collected which carries the information of two side gates and the beam in the middle of them. Fig. 6 shows a typical data trace during the experiment. Here, the positions of the right-side gate and the beam can be clearly seen as sudden peaks indicated with colored circles.

By taking only one input point by the user to define the initial position which a click, the data analysis program scans through the data points and picks up the maximum values for specific intervals, which corresponds to beam and side gates, using the periodicity of the repeating beam and side gate peaks. Even though the beam's peak position changes as the comb drive voltage difference increases, the program still successfully distinguishes whether a peak belongs to the beam or a side gate. After scanning $65 \mathrm{M}$ data points, difference of data points between beam and side gate peaks are stored for varying comb drive voltage levels. The entire operation of the program takes less than 10 seconds.
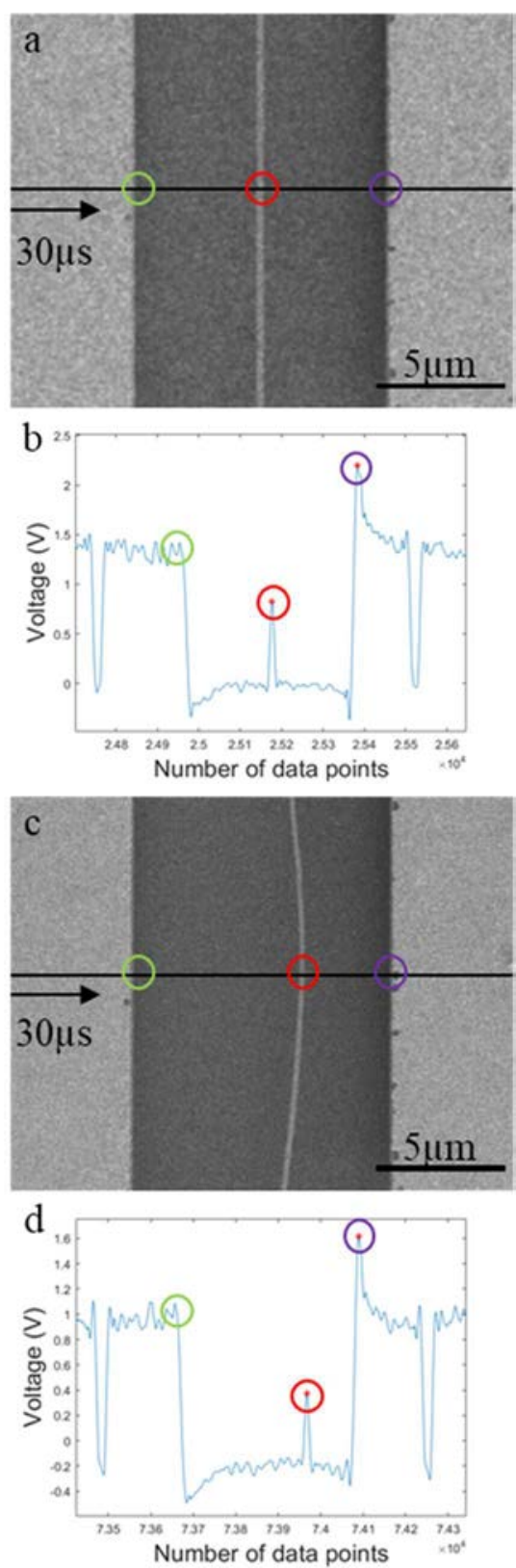

Figure 6: Line mode operation illustrated in the SEM $(a, c)$ and the corresponding raw data $(b, d)$ from the SEM electron detector. In the raw data, the positions of the beam and side gate emerge as peaks. Green, red and purple circles represent left-side gate, beam and right-side gate respectively. Scan line is fixed through the experiment and scan rate is $30 \mu \mathrm{s}$. SEM image and corresponding secondary electron data for $(a, b)$ beam at unbuckled state and $(c, d)$ beam at buckled state.

Fig. 7a shows the deflection of the beam as comb voltage difference increases for $17.5 \mathrm{~V}$ fixed side gate voltage, from non-buckled state to post buckled state. Two data points originating from two distinct input conditions exists at $0 \mathrm{~V}$ at Fig. $7 \mathrm{~b}$. The lower data point near the origin is the starting point for the experiment where both the comb drive voltage difference and side gate voltage is $0 \mathrm{~V}$. The upper data point is achieved when only the side gate is turned on (17.5V for this case), while comb drive voltage is still $0 \mathrm{~V}$. In other words, the upper data point is the effect of pure bending, without any influence of the axial force 
which eventually induces buckling. Through the experiment deflections as low as $15 \mathrm{~nm}$ were detected. Error bars having minimum value of $\pm 7 \mathrm{~nm}$ are recorded which corresponds to noise floor of approximately, $40 \mathrm{pm} / \sqrt{\mathrm{Hz}}$. This is a 250 times more improvement compared to the earlier work with platforms that can fully control nanomechanical buckling [4].
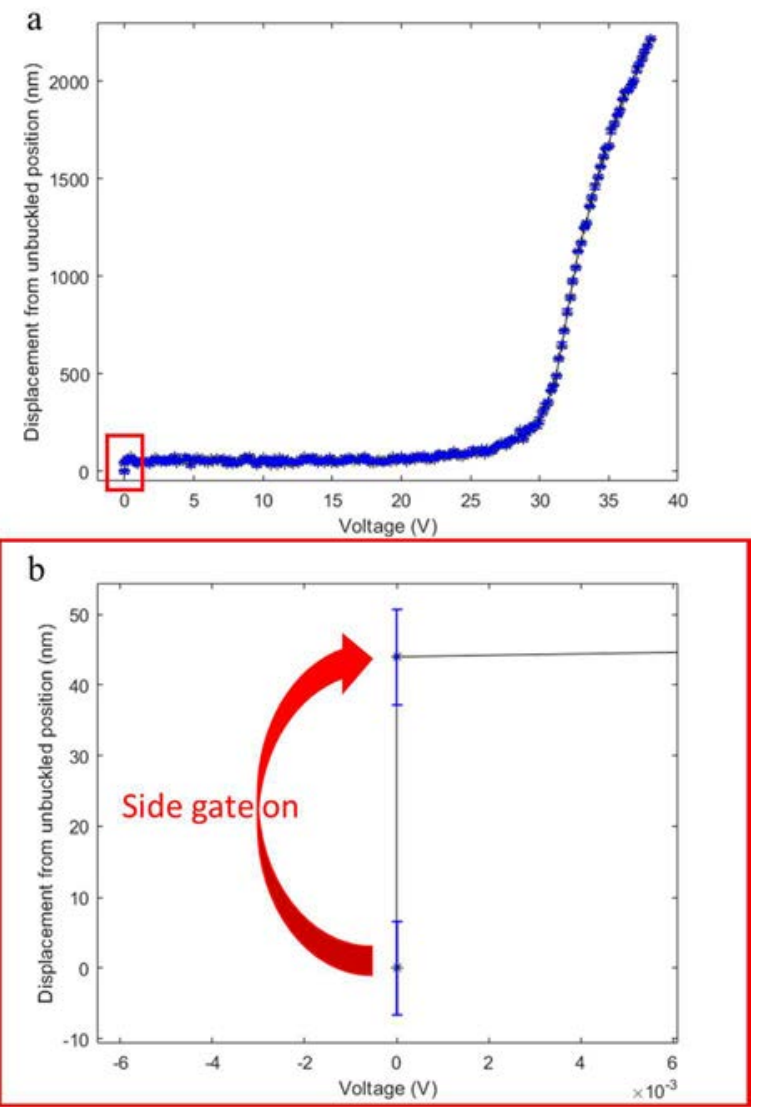

Figure 7: (a) Deflection of the beam starting from 0V to $38 \mathrm{~V}$ comb voltage difference. (b) Deflection of the beam caused by the side gate when its voltage is turned on.

In addition to improving displacement sensitivity, we have also decreased the voltage necessary to induce buckling in these platforms. Previously, a typical value of $45 \mathrm{~V}$ across the comb drive was required to induce buckling. By reducing the device thickness with increasing plasma etching time, the buckling voltage threshold decreased to $12.6 \mathrm{~V}$ (Figure 8). The reduction in buckling voltage is critical since reaching a buckling threshold value of $5 \mathrm{~V}$ will enable this platform to be operated directly by and interfaced with digital electronic systems.

\section{CONCLUSION}

In this work, ultrafast measurement of the buckling behavior, starting from unbuckled position and reaching to large deformations at the post-buckling regime, have been demonstrated. Achieving such accurate measurements with low noise floor is critical to observe snap through characteristics and reveal rich chaotic dynamics arising in the buckling system. We were able to detect deflections with error bar of $\pm 7 \mathrm{~nm}$ with $33 \mathrm{kHz}$ scan frequency using the line mode of SEM. This performance translates into a displacement noise floor levels of $40 \mathrm{pm} / \sqrt{ } \mathrm{Hz}$. Moreover, the buckling voltage levels were reduced by a factor of three compared to previous work. The fine control and simultaneous measurement of the amplitude and direction of this bistable system — without generating significant heat - serves as a new probe for the investigations of nonlinear dynamics at the post-buckling state [5]. The nano-buckling platform can also serve as a candidate mechanism for cavity tuning, evanescent field intensity control and switching applications in optomechanics.

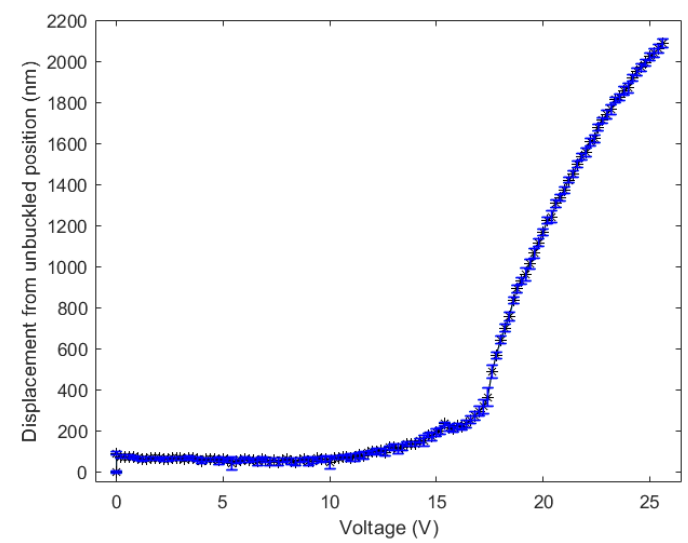

Figure 8: Deflection of the beam starting from $0 \mathrm{~V}$ to $25.6 \mathrm{~V}$ comb voltage difference. Buckling initiation occurs around $12.6 \mathrm{~V}$.

\section{ACKNOWLEDGEMENTS}

This work was supported by the Scientific and Technological Research Council of Turkey (TÜBITAK), Grant No. EEEAG-115E833. The authors thank Michael L. Roukes for useful discussion.

\section{REFERENCES}

[1] D. Roodenburg, J. Spronck, H. Van der Zant, and W. Venstra, "Buckling beam micromechanical memory with on-chip readout," Applied Physics Letters, vol. 94, p. 183501, 2009.

[2] L. Medina, R. Gilat, B. Robert Ilic, and S. Krylov, "Experimental dynamic trapping of electrostatically actuated bistable micro-beams," Applied physics letters, vol. 108, p. 073503, 2016.

[3] B. Charlot, W. Sun, K. Yamashita, H. Fujita, and H. Toshiyoshi, "Bistable nanowire for micromechanical memory," Journal of Micromechanics and Microengineering, vol. 18, p. $045005,2008$.

[4] S. O. Erbil, U. Hatipoglu, C. Yanik, M. Ghavami, A. B. Ari, M. Yuksel, et al., "Full electrostatic control of nanomechanical buckling," Physical review letters, vol. 124, p. 046101, 2020.

[5] M. Gomez, D. E. Moulton, and D. Vella, "Critical slowing down in purely elastic 'snapthrough'instabilities," Nature Physics, vol. 13, pp. 142-145, 2017. 